\title{
INSTRUMENTATION AND CONTROL METHODOLOGY FOR ZERO DEFECT MANUFACTURING IN BORING OPERATIONS
}

\author{
ARSUAGA BERRUETA, M[ikel]; ORTIZ, J[avier]; LOBATO, R[oberto]; \\ FERNANDEZ VALDIVIELSO, A[sier] \& LOPEZ DE LACALLE, L[uis] N[orberto]
}

\begin{abstract}
In this research work an innovative system for quality control of large components boring process is presented in order to get zero defect parts. In traditional manufacturing, the quality control is performed at the end of the manufacturing line so that a defect produced in an initial stage spreads throughout the process. The impact of defective parts in production is particularly important in sectors such as wind energy, aerospace, chemical, etc. due to the large size and high production cost thereof. Moreover, the adjustment of production through statistical process control is inefficient due to the complexity of the parts, the high number of parameters to be controlled and the generally short series.

The new methodology is based on modeling the cutting process and in-line monitoring using telemetry hardware specifically developed and integrated into the boring head. Through readings of different sensors and preliminary process modeling, the system resets the machining strategy and working parameters to correct possible deviations due to tool wear, bending and vibration of mechanical components.
\end{abstract}

Keywords: boring, monitoring, quality control, force model, chatter

\section{INTRODUCTION}

In emerging sectors like chemical, aerospace, etc, where big size parts, with high added value and short production series are manufactured, the End Of Line quality control methodologies such as Six-sigma and SPC have not satisfactory results due to the high economical impact of manufacturing a defective part. In wind turbine industries for example, the quality control inspections becomes critical so they are carried out between the different manufacturing stages, producing increment in the costs and time losses due to movements between measuring and machining machines.

This work proposes a new methodology to approach to the problem solution from two different ways. One of them looks forward the elimination of the predictable defects with the correct adjustment of the process parameters $[1,2]$. The other one goes by the way of inline reworking of the defects that thanks to the monitoring can be detected while machining.

The new technological developments will be based on new measuring tools and sensors able to monitor real time [3] cutting parameters and the later integration of these measurements in the mechanistic models that describe the machining process. By this way, with the combination of manufacturing and verification, it will be possible to minimize the number of defective parts after manufacturing line, reducing costs and verification times. Indirectly, it will be also possible to measure the cutting parameters by the logging of the electrical motors power consumption, but the damping oncoming from the mechanical and electrical assembly will not let us take sufficiently sensitive signals to distinguish defects out of tolerance.

Even this new manufacturing idea can be universally applied to any material removing process, this work is focused on boring operation, very usual in sectors like wind-mill industry, aeronautics, chemical, etc... in the manufacturing of big size and high cost components.

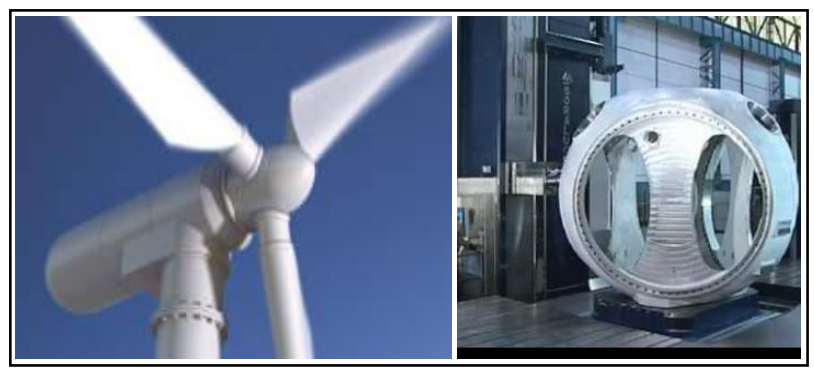

Fig. 1. Big parts manufacturing for wind mill industry

Due to the high operational cost of the big machines used to manufacture big planetary gears for wind mills, the validation work has been done in the mechanical workshop of the E.U.I.T.I. Bilbao using a Kondia B700 milling machine equipped with a D'Andrea TA-125 boring head.

\section{CONTROL LOOP}

The traditional way to make the machining program is to predict the cutting parameters under some standard calculations, to adjust them after some machined parts in a trial and error process. After the manufacturing line, the quality control is made over the part and the possible deviations detected on the measuring process are studied to make the needed corrections on the manufacturing parameters. For example in some components of the wind mill gear boxes, the check control is made between different manufacturing steps of the same component to avoid error propagation between different stages. To ensure that each machining operation is well done becomes necessary. These manufacturing stops become really expensive and in some of the cases in which the component needs to be moved to other place to be measured, for example a Coordinate Measuring Machine, these costs become even more critical. Anyway, it continues to be economically acceptable to make all those mid-process verifications to avoid defective parts.

In the proposed new manufacturing methodology, the manufacturing and the control take place at the same 
machine while the material is being removed. To achieve this objective two different control loops are defined. The first one uses the deformation of the boring bar which takes the information out coming from the strain gauge. The second one measures the vibrations using an accelerometer. Both are shown in Fig. 2 in yellow and brown colors respectively.

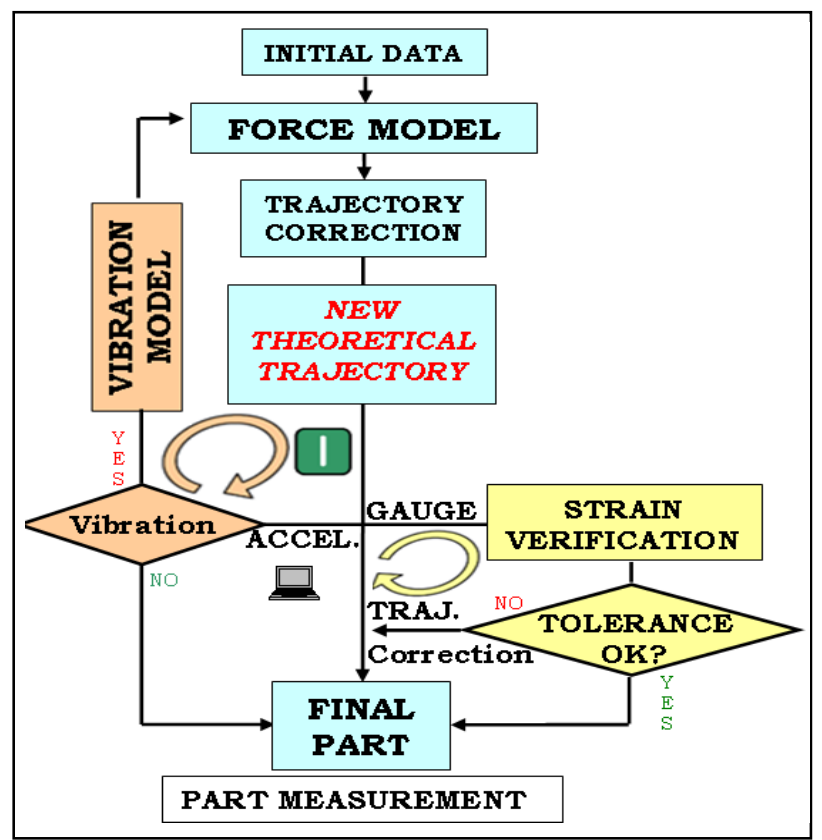

Fig. 2. Flowchart of the methodology proposed

Even the rigidity of the machines usually used on these processes are very high, same are the manufacturing requirements. Because of that it is very important to measure the small deflections in the boring bar that produce the displacement of the tool tip and error in the manufactured part. For this issue a previously mounted on the bar and calibrated strain gauge has been used. The forces on the tool tip are transmitted to the model by the gauge signal [4], and are compared with the theoretical forces. The difference between both forces will be proportional (stiffness) to the deflection of the bar. Knowing the deflection of the bar it is possible to correct in real time the trajectory of the tool in the same value.

The vibrations on the tool tip are measured to detect the non stable machining regions. If the vibration exceeds some levels, the part will be produced with bad surface quality. To achieve expected roughness in the surface, the model will vary the cutting conditions $\left(\mathrm{v}_{\mathrm{c}}, \mathrm{f}\right.$ and $a_{p}$ ) when an excessive vibrations level is detected, to get back the stable machining.

\section{METHODS AND EQUIPMENT}

\subsection{Experimental equipment}

The experimental validation of the developments proposed in this work has been done using an OROS OR35 (NVGate Software) signal analyzer and a 086C03 impact hammer. 333B32 accelerometers have been used for the characterization of the modal parameters of the boring head and the embedded accelerometers on the tool tip. The statical calibration of the strain gauge for the three cutting force directions has been made with a PCE FG-5K dynamometer.

The previous design of the gauge and its corresponding position on the boring tool has been made with Unigraphics NX7.5.

\subsection{Design of data acquisition and telemetry tooling}

The hardware is based on the nanowatt series low energy 18LF4620 Microcontroller with $8 \mathrm{~Kb}$ Ram memory and 10 bits ADC conversion channels for the gauge and accelerometer measurements logging. It has high processing speed so it can achieve $1 \mathrm{MHz}$ maximum analogical reading frequency. The hardware communicates with the low energy CC1100 radiofrequency transceptor which has a maximum transmission power of $10 \mathrm{dBm}$ and a maximum data transmission speed of $500 \mathrm{KBaudio}$ on high processing speed. For the data storage it is equipped with a $16 \mathrm{Mbit}$ Flash memory.

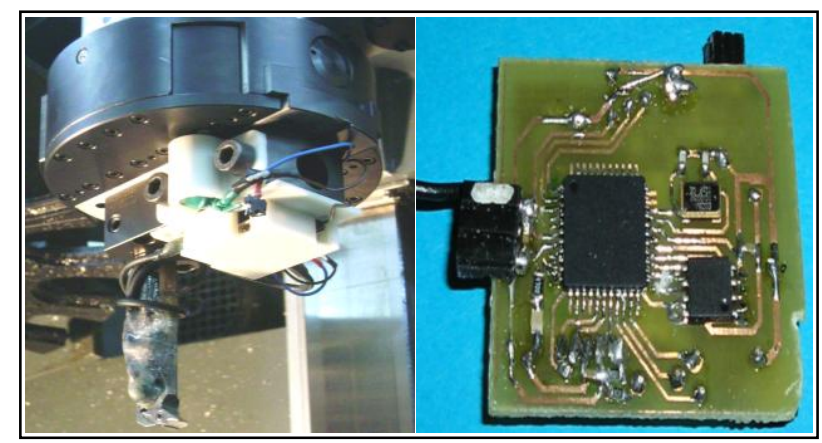

Fig. 3. Data acquisition and telemetry chip mounted in the boring head

The signal from the gauge is configured on Wheatstone bridge and is regulated with the MAX 4208 instrumentation amplificatory and the MAX 9916 operational for the filter and amplification functions. The system is feed by a 3,6V lithium cell in conjunction with an LDO stabilizer for adjust the output voltage to $3,3 \mathrm{~V}$.

\section{STATIC MODEL}

To define the effect of the cutting forces on the boring bar deformation [5], strain gauge sensibility characterization tests have been done independently for the three cutting forces involved.

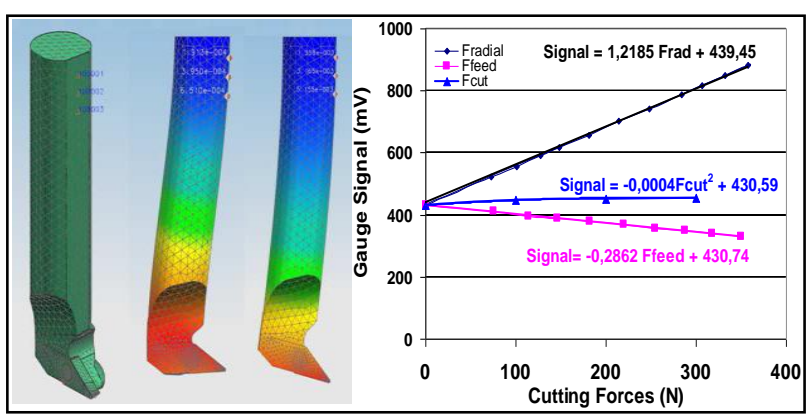

Fig. 4. Previous FEM analysis and experimental deformation set up

The signal of the gauge due to the sum of the three cutting forces $\left(\mathrm{F}_{\mathrm{R}}, \mathrm{F}_{\mathrm{C}}, \mathrm{F}_{\mathrm{F}}\right)$ can be represented as (1) where $S_{R}, S_{C}, S_{C}$, and $S_{F}$, are the sensitivities for each force and effect and $\mathrm{G}_{0}$ the gain offset (experimental values $\mathrm{S}_{\mathrm{R}}=1.218, \mathrm{~S}_{\mathrm{C}}{ }^{\prime}=0.000398, \mathrm{~S}_{\mathrm{C}}=0.197, \mathrm{~S}_{\mathrm{F}}=0.286$, $\mathrm{G}_{0}=430$ ): 


$$
G=S_{R} \cdot F_{R}-S_{F} \cdot F_{F}-S_{C}^{\prime} \cdot F_{C}^{2}+S_{C} \cdot F_{C}+G_{0}
$$

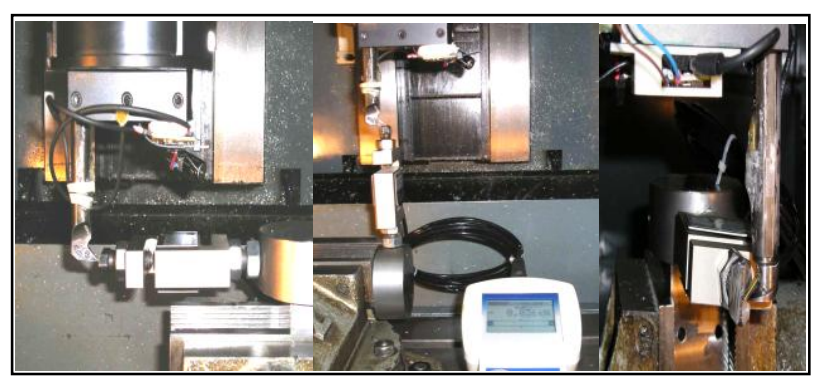

Fig. 5. Gauge sensitivity measurement test on three orthogonal directions

From the material and the cutting forces characterization, it is possible to solve the relation between the three cutting forces, which is approximately constant in the working region and follows the relation (2) and (3) (experimental values $R_{\mathrm{FF}}=0,5786$ $\left.\mathrm{R}_{\mathrm{FC}}=0,3543\right)$. Applying these relations on the equation (1), it is possible to simplify it as (4):

$$
\begin{gathered}
R_{F_{F}}=\frac{F_{R}}{F_{F}} \rightarrow F_{F}=F_{R} / R_{F_{F}} \\
R_{F_{C}}=F_{R} / F_{C} \rightarrow F_{C}=F_{R} / R_{F_{C}} \\
G=S_{R} \cdot F_{R}-S_{F} \cdot F_{R} / R_{F_{F}}-S_{C} \cdot\left(F_{R} / R_{F_{C}}\right)^{2}+S_{C} \cdot F_{R} / R_{F_{C}}+G_{0}
\end{gathered}
$$

To determine the deviation on the tool tip because of the cutting forces measured with the gauge, the static force-deformation model [6] have been used:

$$
\delta_{T T}=\frac{F_{R}}{0,60 \mathrm{e}^{-3}}+\frac{q_{\text {Cenrifi. }}}{1,68}+\frac{q_{\text {Centrif. }} \cdot 75+F_{R}}{4,67}+\frac{\left(0,5 \cdot q_{\text {Centrif }}+F_{R}\right) \cdot 75}{1000 \cdot 0,75}
$$

The stiffness of boring head, bar and milling machine has been measured using an infinite stiffness tool for the decomposition of the cutting and bending moment effects.

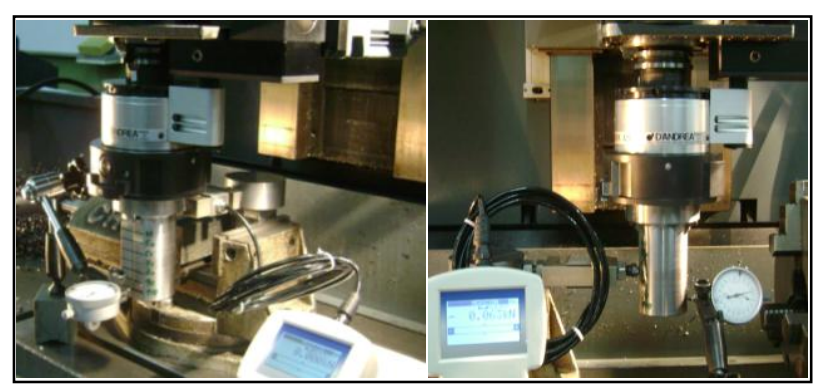

Fig. 6. Whole assembly stiffness characterization

\section{DYNAMICAL STABILITY}

Because of the low dynamical stiffness of the boring bar [7-9], it is essential to achieve a good quality boring operation to avoid vibrations during machining. The vibrations on the tool tip, result in an early tool wear and machine component damages [10] (bearings, gear, etc) from the process point of view, and on the other hand, in the machined part, bad looking surfaces. To be able to identify during machining the stable cutting conditions, the boring bar has been equipped with an accelerometer.

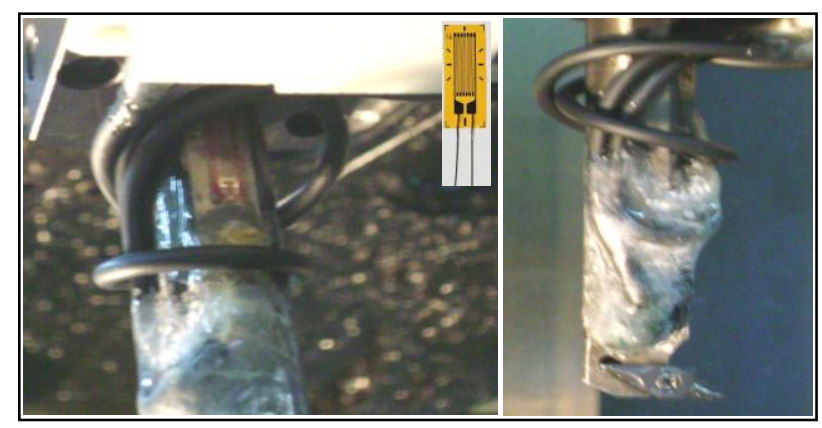

Fig. 7. Detail of the Gauge and Accelerometer embedded in the boring bar

Taking in to account that the effect on the surface finish produced by the vibrations on the cutting force direction is negligible and that the rigidity of the assembly on the feed direction is really high, the accelerometer will be set to measure the vibrations in the radial direction near the tool tip.

\subsection{Static and centrifugal calibration of the accelerometer}

For the validation of the embedded accelerometer before integrating it in the boring bar, some test has been carried out in a cantilever beam tool, to compare the measurements with a commercial accelerometer. Varying the modal characteristics of the tool, several vibration conditions have been tested in the range of the accelerometer. The results of the accelerometer proposed have been matched well with the commercial ones. In Fig. 8 the tool and principal modes are showed.

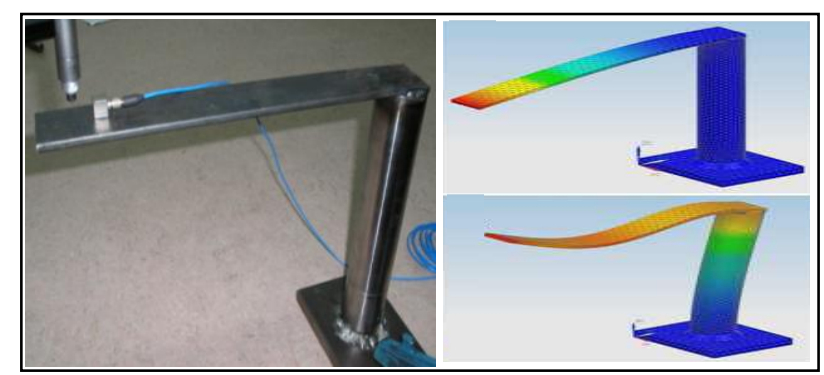

Fig. 8. Static calibration tool

In order to correct the offset in the accelerometers signal produced by the centrifugal forces, different rotating test (radial position and rotating speed combination) have been done with out removing material. Some results can be seen in the Fig. 9.

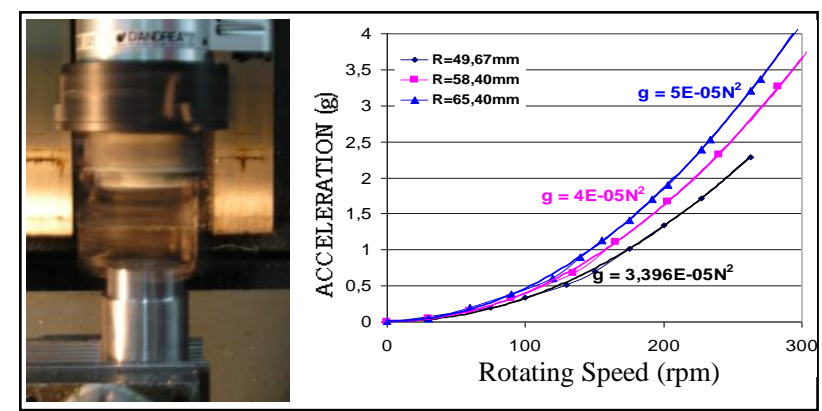

Fig. 9. Accelerometer response for the centrifugal force

Taking in to account the high range of the accelerometer $(70 \mathrm{~g})$ selected to avoid the saturation at 
high rotational speeds, the results have been very good with errors less than $5 \%$ in the worst conditions (minimum rotation radius and speed). The gain error in the usual range of work (cutting speeds more than $100 \mathrm{~m} / \mathrm{min}$ ) is negligible.

While machining to be able to identify the nature (forced, self excited...) of the vibration [11,12], a dynamic characterization of the boring head and bar have been done using an impact hummer. The dominant frequencies are $1800 \mathrm{~Hz}$ for the bar and $300 \mathrm{~Hz}$ for the head approximately. In the machining experiments, acquisition frequencies up to $5 \mathrm{KHz}$ will be necessary.

\section{RESULTS ON MACHINED PART}

In the machining tests, cutting forces, deflections and vibrations have been measured in a test tube for several cutting conditions. In Fig. 10, an image of the boring head rotating at $800 \mathrm{rpm}$ (depth of cut of $0.5 \mathrm{~mm}$ ) and the vibrations signal acquired by the accelerometer are showed.

Under certain cutting conditions, vibrations in machining have been detected, resulting in poorly finished surfaces. Self-excited vibrations (Chatter) have not been sampled in any case along the experimental validation. Except in extremely severe cutting conditions, the gauge signal is not overloaded and the radial force is measured correctly. The tool tip displacements measured were less than $0.06 \mathrm{~mm}$.

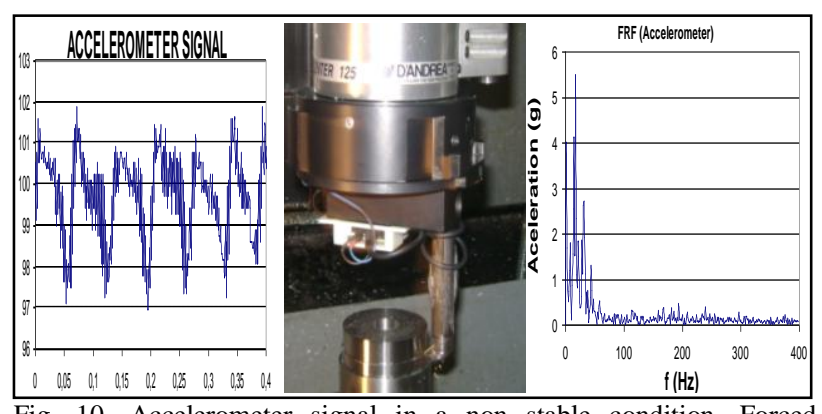

Fig. 10. Accelerometer signal in a non stable condition. Forced vibration case

\section{CONCLUSION}

From the results of the experiments it can be concluded that it is feasible to monitor in-line cutting forces accurately using the telemetry device proposed. Although the force measurements made with a single gauge is correct, would be of great interest to complete these measurements with the reading of a second gauge as it is seen that the sensitivity of the boring bar against the feed force is important and not always constant the relationship between the two main forces. If the differences between measured and theoretical forces are analyzed and the correlation with the dimensional error in the workpiece, it is concluded that the loop control parameter reflect accurately the phenomenon of deformation of the boring bar and its result in the machined part.

Regarding the vibrations measured, the accelerometer has correctly picked vibrations produced in the bar, and as expected, has been observed that in the greatest intensity vibration sequences, the surface of the workpiece has been marked.
Therefore, the work presented shows that the applied procedure is valid for inline control of the boring process and as future work arise, adding to the set and validation of a new gauge in order to refine the extent of the feed force, improved data acquisition in order to sample at higher frequencies and the study of self-excited vibrations to optimize the vibration model.

\section{ACKNOWLEDGEMENTS}

The work presented in this paper was supported within the project entitled MuProD- Innovative Proactive Quality Control System for In-Process Multi-Stage Defect Reduction- of the Seventh Framework Programme of the European Union [FoF.NMP.2011-5] and UPV/EHU under program UFI 11/29.

\section{REFERENCES}

[1] L.N. López de Lacalle, J.A. Sánchez, A. Lamikiz (2004). High Performance Machining, Ed. Izaro, ISBN 978-1-84800-379-8, Bilbao, Spain

[2] F.J. Campa (2009). Metodología para la predicción de la Estabilidad Dinámica en el Mecanizado de Alta Velocidad de suelos delgados, Ph.D. Dissertation, Mechanical Engineering, University of The Basque Country, Bilbao, Spain

[3] R. Teti, K. Jemielniak, G. O'Donnell, D. Dornfeld (2010). Advanced monitoring of machining operations, CIRP Annals Manufacturing Technology 59, 717-739

[4] M. Milfelner, F. Cus, J. Balic (2005), An overview of data acquisition system for cutting force measuring and optimization in milling, Journal of Materials Processing Technology 164-165 (2005) 1281-1288

[5] G. Totis, G. Wirtz, M. Sortino, D. Veselovac, E. Kuljanic, F. Klock (2010)., Development of a dynamometer for measuring individual cutting edge forces in face milling, Mechanical Systems and Signal Processing, 24, 1844-1857

[6] M. Arsuaga, L.N. López de Lacalle, R. Lobato, G. Urbikain, F. Campa (2012). Force and Deformation Model for Error Correction in Boring Operations. International Journal of Machine Tools and Manufacture, Advanced Materials Research, Vol. 498 (2012) pp 121-126

[7] F. Atabey, I. Lazoglu, Y. Altintas (2003). Mechanics of boring processes-Part I. International Journal of Machine Tools and Manufacture, Advanced Materials Research, Volume 43, Issue 5, April 2003, Pages 463-476

[8] B. Moetakef-Imani, N.Z.Yussefian (2009). Dynamic simulation of boring process. International Journal of Machine Tools \& Manufacture 49 (2009)1096

[9] H. Akesson, T. Smirnova,L Hakansson (2009), Analysis of dynamic properties of boring bars concerning different clamping conditions, Mechanical Systems and Signal Processing 23 (2009) 2629-2647

[10] Altintas Y. (2000). Manufacturing automation: metal cutting, mechanics, machine tool vibrations, and CNC design., Cambridge University Press, Cambridge, UK

[11] E. Budak, E. Ozlu (2007). Analytical Modeling of Chatter Stability in Turning and Boring Operations: A Multi-Dimensional Approach, CIRP Annals - Vol. 56/1/2007

[12] C. Mei (2005), Active regenerative chatter suppression during boring manufacturing process, Robotics and Computer-Integrated Manufacturing 21 (2005) 153-158 